\title{
THE
}

4-1-1991

\section{Field-Induced Kosterlitz-Thouless Transition in the Zero- Temperature Triangular Ising Antiferromagnet}

\author{
H. W.J. Blöte \\ M. P. Nightingale \\ University of Rhode Island, nightingale@uri.edu
}

X. N. Wu

A. Hoogland

Follow this and additional works at: https://digitalcommons.uri.edu/phys_facpubs

Terms of Use

All rights reserved under copyright.

\section{Citation/Publisher Attribution}

Blöte, H. W. J., Nightingale, M. P., Wu, X. N., \& Hoogland, A. (1991). Field-induced Kosterlitz-Thouless

transition in the zero-temperature triangular Ising antiferromagnet. Physical Review B, 43(10), 8751-8754. doi: $10.1103 /$ PhysRevB.43.8751

Available at: http://dx.doi.org/10.1103/PhysRevB.43.8751

This Article is brought to you for free and open access by the Physics at DigitalCommons@URI. It has been accepted for inclusion in Physics Faculty Publications by an authorized administrator of DigitalCommons@URI. For more information, please contact digitalcommons-group@uri.edu. 


\title{
Field-induced Kosterlitz-Thouless transition in the zero-temperature triangular Ising antiferromagnet
}

\author{
H. W. J. Blöte \\ Laboratorium voor Technische Natuurkunde, Technische Universiteit Delft, Postbus 5046, \\ 2600 GA Delft, The Netherlands \\ M. P. Nightingale \\ Physics Department, University of Rhode Island, Kingston, Rhode Island 02881 \\ X. N. Wu \\ The Rockefeller University, 1230 York Avenue, New York, New York 10021-6399 \\ A. Hoogland \\ Laboratorium voor Technische Natuurkunde, Technische Universiteit Delft, Postbus 5046, \\ 2600 GA Delft, The Netherlands
}

(Received 28 November 1990)

\begin{abstract}
We investigate the zero-temperature triangular Ising antiferromagnet in a magnetic field by means of transfer matrix and Monte Carlo methods. The finite-size results are compared with predictions obtained from a mapping to the Gaussian model. The results confirm the presence of a field-induced Kosterlitz-Thouless transition to a state with long-range order.
\end{abstract}

The ground state of the antiferromagnetic triangular Ising model with only nearest-neighbor interactions is characterized by the absence of long-range order and has a nonzero entropy, ${ }^{1}$ at least in the isotropic case. Since the correlation functions decay with power laws of the distance $^{2}$ rather than exponentially, the ground state is critical. When a magnetic field $B$ of sufficient strength is applied to the ground state, the residual entropy is removed. The resulting ordered state has spins of one sign on two of the three sublattices of the triangular lattice, and spins of the opposite sign on the third sublattice. Here we investigate the nature of the associated phase transition, induced by a magnetic field of the order of the (vanishing) thermal energy $k T$. Thus, we consider the $T \rightarrow 0$ limit such that $H=B / k T$ remains finite, while the nearest-neighbor coupling $K$ does not: $K=J / k T \rightarrow-\infty$. The reduced Hamiltonian is

$$
\mathcal{H} / k T=-K \sum_{<i, j>} s_{i} s_{j}-H \sum_{k} s_{k}
$$

where the first sum is over all nearest-neighbor pairs. The net effect of this sum is, apart from contributing an infinite constant, the restriction that elementary triangles with three spins of the same sign are forbidden.

It was originally suspected ${ }^{3}$ that the reduced field $H$ was relevant, i.e., that the system would immediately enter the ordered state when $H \neq 0$. However, this was not supported by a later analysis ${ }^{4}$ which used a mapping to the Gaussian model and the Coulomb gas. ${ }^{5}$ We summarize some of the main steps of this analysis. As a first step, the zero-temperature Ising model is exactly mapped to the triangular Ising solid-on-solid (TISOS) model, ${ }^{6}$ a solid-on-solid (SOS) model describing the equilibrium shape of a (111) surface of a cubic crystal. ${ }^{4} \mathrm{Us}$ ing this mapping, one can express the SOS height-height correlation function $G(r)=\left\langle\left(h_{r}-h_{0}\right)^{2}\right\rangle$, between height variables $h_{r}$ and $h_{0}$ separated by a distance $r$, in correlations between Ising spins. The latter correlations are known exactly for $H=0 .{ }^{2,4}$ The asymptotic behavior of $G(r)$ at long distances is thus found to be

$$
G(r) \simeq \frac{9}{\pi^{2}} \ln r
$$

Such logarithmic divergences are typical for twodimensional Gaussian 7,8 and SOS models in their rough phases. ${ }^{9}$ In the Gaussian model, with a reduced Hamiltonian $-\left(2 \pi / T_{\mathrm{G}}\right) \sum_{<i, j\rangle}\left(h_{i}-h_{j}\right)^{2}$, the amplitude $A$ of the logarithm can be calculated as a function of the Gaussian temperature $T_{\mathrm{G}}$ as $A=T_{\mathrm{G}} /\left(2 \pi^{2}\right)$. It is plausible that under a renormalization-group transformation the TISOS model flows to the Gaussian model. Since the amplitude of the logarithm is invariant under this transformation, it follows that the TISOS model maps onto a Gaussian model with $T_{\mathrm{G}}=18$. Consequently, we know the critical-point exponents of the TISOS and equivalent Ising model. As an example we consider the critical exponent of the uniform magnetic field $H$ coupling to the Ising spins. As argued in Ref. 4, this field corresponds to a periodic potential with a period of $p=2$ elementary steps of the SOS surface. In the Gaussian model the 
corresponding contribution to the Hamiltonian is a term proportional to $\cos \left(2 \pi h_{i} / p\right)$, with a scaling dimension ${ }^{8}$

$$
X_{p}=T_{\mathrm{G}} / 2 p^{2}
$$

which is equal to $\frac{9}{4}$ for $p=2$. This scaling field is irrelevant: If sufficiently small, it does not destroy the algebraic correlations in the zero-field Ising ground state (at least, if our assumption concerning the existence of a mapping between the SOS and the Gaussian model was right). However, it is also to be expected ${ }^{4}$ that $H$ will play the role of a temperaturelike parameter and will decrease the marginal Gaussian temperature field. At some value $H_{\mathrm{KT}}$ the scaling dimension $X_{2}$ will become marginal $\left(T_{\mathrm{G}}=16\right)$, and the system will undergo a Kosterlitz-Thouless (KT) transition. ${ }^{10}$ Another consequence of this theory is that the smallest anomalous dimension is $X_{6}$, which is equal to $\frac{1}{4}$ at $H=0 ; X_{6}$ is expected to decrease as a function of $|H|$ and should reach the value $\frac{2}{9}$ at $H=H_{\mathrm{KT}}$. For $H>H_{\mathrm{KT}}$ the system enters a long-range ordered state which is threefold degenerate: the majority of the minus spins are located on one of the three sublattices of the triangular lattice. The anomalous dimension $X_{6}$ is associated with a staggered field, ${ }^{4}$ e.g., $-2 H$ on one sublattice and $+H$ on the other two sublattices, and should govern the long-range behavior of the spin-spin correlation function $g(r) \simeq a r^{-2 X_{6}}$.

In this paper we verify this scenario by two different approaches. Firstly, we determine the magnetic susceptibility of the zero-temperature Ising model as a function of the magnetic field for several finite $L \times L$ systems with toroidal boundaries. Finite-size scaling predicts that the susceptibility $\chi(L)$ of a system with linear size $L$ at a critical point scales as

$$
\chi(L) \simeq \chi(0)+A L^{2 y_{H}-2}+\cdots,
$$

where $A$ is a constant and the ellipsis stands for corrections to scaling and $y_{H}$ is the renormalization exponent associated with the field $H$. If the transition is indeed $\mathrm{KT}$-like, $y_{H}=0$ and the susceptibility will not diverge; it will behave qualitatively as the specific heat of the $X Y$ model. However, if the transition is of the three-state Potts type, as might be suggested by the symmetry of the ordered state, we have $y_{H}=\frac{6}{5}$, so that $\chi(L)$ will diverge with $L$.

Figure 1 shows Monte Carlo results for the susceptibility obtained by means of the Delft Ising System Processor (DISP) (Ref. 11) for systems with linear dimensions $L$ equal to powers of 2 , ranging from 8 to 256 . The simulations were performed at $K=-12$, which, after truncation of the Monte Carlo transition probabilities to machine precision, inhibits spin flips that increase the nearest-neighbor interaction energy.

The way in which the initial states were prepared deserves some attention. Via the SOS representation, a $T=0$ Ising configuration can be specified by means of a system of nonintersecting strings. ${ }^{6}$ Since the number of strings is conserved under the Glauber ${ }^{12}$ dynamics used in the spin-updating algorithm, it should initially be set

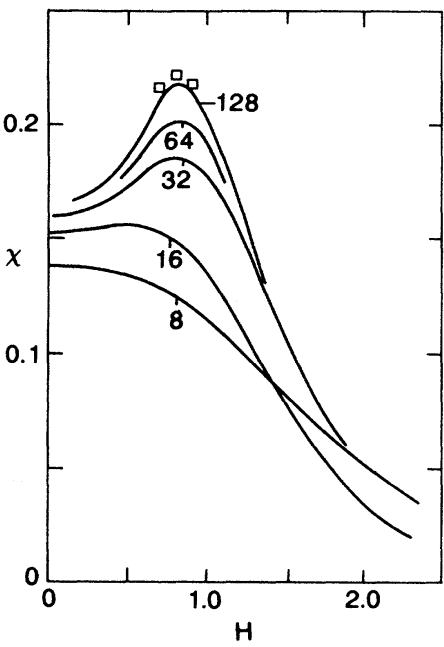

FIG. 1. Monte Carlo results for the magnetic susceptibility $\chi$ of the zero-temperature triangular Ising model with a finite coupling $H$ of the spins to the magnetic field. Results are shown for several $L \times L$ systems with periodic boundaries and $L$ equal to powers of 2 . In order to avoid crowding of symbols, the data are shown by smooth curves except for the largest system size (256) for which the data points are shown as $\square$. The statistical error in the latter points is slightly larger than the size of the symbols. The errors in the other data do not exceed the thickness of the curves in most cases. The susceptibilities for system sizes $L=2$ and 4 are zero because the initial state (described in the text) is frozen: no spin flips are possible. These results shown here indicate that the susceptibility remains finite when the system enters the ordered phase.

equal to $2 L / 3$ which is appropriate for the state at $H=0$ as well as the ordered state at $0 \ll H \ll-6 K$. It does not seem likely that fluctuations in the number of strings, as occurring in the summation over all Ising configurations in the partition sum, would alter universal aspects of the critical behavior. Since the DISP can only simulate systems with sizes equal to powers of 2 , the aforementioned choice is not possible and we have chosen the number of strings equal to the nearest even integer to $2 L / 3$ instead. Only configurations with an even number of strings correspond to Ising configurations with periodic boundaries. This choice leads to corrections to scaling in $\chi$ proportional to $1 / L$, and to some alternation between results for system sizes that are odd and even powers of 2. While such corrections to scaling, and alternation, are much in line with the data shown in Fig. 1, there are no indications for a power-law divergence of $\chi(L)$ with $L$.

As a further check of the Gaussian model description of the $T=0$ Ising model, we have performed a finitesize scaling analysis ${ }^{13,14}$ using transfer matrix calculations. These calculations pertain to a lattice wrapped on an infinitely long cylinder, with one set of lattice edges perpendicular to the cylinder's axis. The finite-size parameter $L$ is the circumference of the cylinder measured in lattice units. 
The transfer matrix $T$ that adds two layers of $L$ spins to the lattice can be written as

$$
\underline{T}=\underline{T}_{H}^{1 / 2} \underline{T}_{K+} \underline{T}_{H} \underline{T}_{K}-\underline{T}_{H}^{1 / 2}
$$

where the diagonal matrix $\underline{T}_{H}$ takes into account the field $H$ acting on a row of spins. It is defined by

$$
T_{H}\left(\mathbf{s}, \mathbf{s}^{\prime}\right)=\prod_{i=1}^{L} \exp \left[H\left(s_{i}+s_{i}^{\prime}\right)\right] \delta_{s_{i}, s_{i}^{\prime}}
$$

where rows of spins are denoted as $\mathbf{s}=\left(s_{1}, s_{2}, \ldots, s_{L}\right)$. The matrices $\underline{T}_{K \pm}$ take into account half the couplings within a row, and the couplings between neighboring rows of spins:

$$
\begin{aligned}
T_{K \pm}\left(\mathbf{s}, \mathbf{s}^{\prime}\right)=\prod_{i=1}^{L} \exp \{K[ & s_{i}^{\prime}\left(s_{i}+s_{i \pm 1}\right) \\
& \left.\left.+\left(s_{i} s_{i+1}+s_{i}^{\prime} s_{i+1}^{\prime}\right) / 2\right]\right\} .
\end{aligned}
$$

Since $\underline{T}_{K_{-}}$is the transpose of $\underline{T}_{K+}, T$ is symmetric. The matrices $\underline{T}_{K+}$ and $\underline{T}_{K-}$ can be decomposed into $L$ sparse matrices, which is convenient for computational purposes. ${ }^{15,13}$ This decomposition proceeds as for the honeycomb lattice, see, e.g., Ref. 16. However, since we are now working in the limit $K \rightarrow-\infty$, we have to be careful to share each bond strength $K$ between adjacent triangles and to divide out an infinite factor $\exp (-K / 2)$ from the Boltzmann weight of each triangle.

The correlation length can be expressed in the two largest eigenvalues $\lambda_{1}$ and $\lambda_{2}$ of the transfer matrix as

$$
\xi(H, L)=1 /\left[\zeta \ln \left(\lambda_{1} / \lambda_{2}\right)\right]
$$

where the geometrical factor $\zeta$ equals $1 / \sqrt{3}$. According to the theory of conformal invariance ${ }^{17}$ the asymptotic finite-size scaling behavior of the correlation length of a critical system obeys

$$
\lim _{L \rightarrow \infty} L / \xi(H, L)=2 \pi X,
$$

where $X$ is the scaling dimension of the correlation function associated with the correlation length. Hence, the scaled gaps $X(H, L)=L /[2 \pi \xi(H, L)]$ should converge to the scaling dimension $X$.

Figure 2 shows the scaled gaps as a function of $H$ for several system sizes that are a multiple of 3 . At $H=0$, the data points converge well to the expected value $X_{6}=\frac{1}{4}$ [see Eq. (3)] for the smallest anomalous dimension, also in agreement with the correlation function exponent found by Stephenson. ${ }^{2}$ For not too large values of $H \neq 0$, the data points still show convergence to a smaller but nonzero value of $X$ for $L \rightarrow \infty$, thus revealing a decrease of the corresponding Gaussian temperature $T_{\mathrm{G}}$. For $H$ larger than about 0.3 the data points

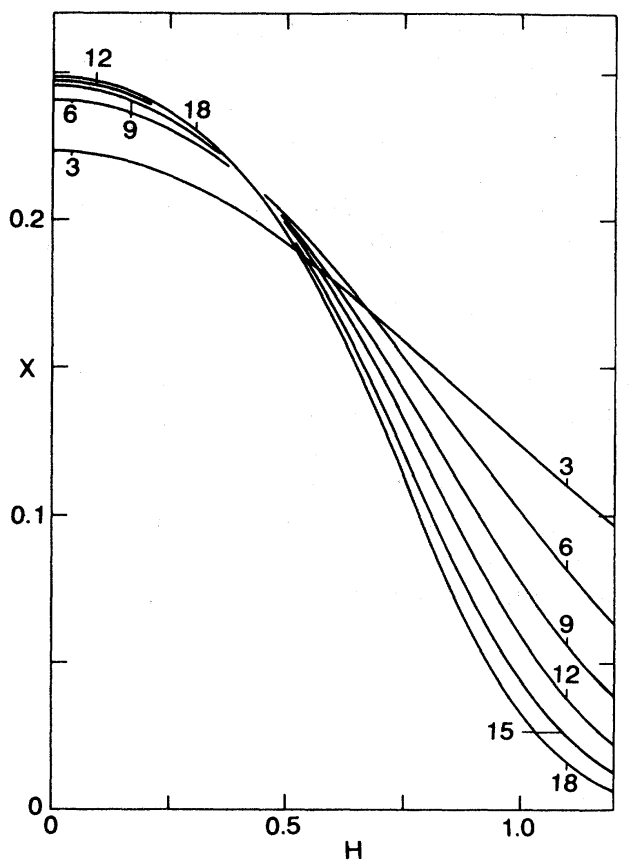

FIG. 2. Scaled gaps $X$ of $L \times \infty$ zero-temperature antiferromagnetic triangular Ising models, as a function of the reduced magnetic field $H$. Data points (not shown) were calculated at intervals of 0.2 in $H$ and connected by smooth curves. Each curve is labeled by the value of the finite size $L$. These data show that $X$ converges to a nonzero value when $H$ is smaller than about 0.3 or 0.4 . For large values of $H$ we observe that $X$ converges to 0 with increasing $L$. Such behavior is in agreement with the presence of a Kosterlitz-Thouless transition to the ordered state.

assume a clearly decreasing behavior with $L$. Convergence to $X=0$ is observed for larger values of $H$, in agreement with the behavior expected for a long-range ordered state (an infinite correlation length). The decreasing trend sets in at a value of $X$ close to $\frac{2}{9}$ : the value predicted for $X_{6}$ at the $\mathrm{KT}$ transition. We conclude that these results provide strong evidence for a field-induced $\mathrm{KT}$ transition at about $H=0.3$ in the zero-temperature antiferromagnetic triangular Ising model.

We are indebted to Professor B. Nienhuis for valuable discussions, and to P.A.M. Cornelissen for assistance during the Monte Carlo calculations. The hospitality of Professor E.G.D. Cohen at the Rockefeller University in New York, where some of this work was performed, is gratefully acknowledged. This research is supported in part by the (U.S.) National Science Foundation through Grant No. DMR-87-04730 and by NATO Grant No. 198/84. 
${ }^{1}$ R.M.F. Houtappel, Physica 16, 425 (1950).

${ }^{2}$ J. Stephenson, J. Math. Phys. A 11, 413 (1970).

${ }^{3}$ W. Kinzel and M. Schick, Phys. Rev. B 23, 3435 (1981).

${ }^{4}$ B. Nienhuis, H.J. Hilhorst, and H.W.J. Blöte, J. Phys. A 17, 3559 (1984).

${ }^{5}$ B. Nienhuis, in Phase Transitions and Critical Phenomena, edited by C. Domb and J.L. Lebowitz (Academic, New York, 1987), Vol. 11.

${ }^{6}$ H.W.J. Blöte and H.J. Hilhorst, J. Phys. A 15, L631 (1982).

${ }^{7}$ H.J.F. Knops, Phys. Rev. Lett. 39, 766 (1977).

${ }^{8}$ J.V. José, L.P. Kadanoff, S. Kirkpatrick, and D. Nelson, Phys. Rev. B 16, 1217 (1977).

${ }^{9}$ Henk van Beijeren, Phys. Rev. Lett. 38, 993 (1977).

${ }^{10}$ J.M. Kosterlitz and D.J. Thouless, J. Phys. C 5, L124 (1973); 6, 1181 (1973).

${ }^{11}$ A. Hoogland, J. Spaa, B. Selman, and A. Compagner, J.
Comp. Phys. 51, 250 (1983); A. Hoogland, A. Compagner, and H.W.J. Blöte, in Special Purpose Computers, edited by B.J. Alder, Vol. 5 of Computational Techniques (Academic, New York, 1988).

${ }^{12}$ R.J. Glauber, J. Math. Phys. 4, 294 (1963).

${ }^{13}$ For reviews of finite-size analysis, see M.P. Nightingale, J. Appl. Phys. 53, 7927 (1982); and in Finite-Size Scaling and Numerical Simulation of Statistical Systems, edited by V. Privman (World Scientific, Singapore, 1990).

${ }^{14}$ M.N. Barber, in Phase Transitions and Critical Phenomena, edited by C. Domb and J.L. Lebowitz (Academic, New York, 1983), Vol. 8.

${ }^{15}$ M.P. Nightingale, Proc. K. Ned. Akad. Wet. Ser. B 82, 235 (1979).

${ }^{16}$ H.W.J. Blöte, F.Y. Wu and X.N. Wu, Int. J. Mod. Phys. B 4, 619 (1990).

${ }^{17}$ J.L. Cardy, J. Phys. A 17, L385 (1984). 